\title{
On the Uphill Domination Polynomial of Graphs
}

\author{
Thekra Alsalomy, Anwar Saleh, Najat Muthana, Wafa Al Shammakh \\ Department of Mathematics, Faculty of Science, University of Jeddah, \\ Jeddah, Saudi Arabia \\ Email: talsalomy.stu@uj.edu.sa,math.msfs@gmail.com,nmuthana@uj.edu.sa,wsalshamahk@uj.edu.sa
}

How to cite this paper: Alsalomy, T., Saleh, A., Muthana, N. and Al Shammakh, W. (2020) On the Uphill Domination Polynomial of Graphs. Journal of Applied Mathematics and Physics, 8, 1168-1179. https://doi.org/10.4236/jamp.2020.86088

Received: April 20, 2019

Accepted: June 20, 2020

Published: June 23, 2020

Copyright $\odot 2020$ by author(s) and Scientific Research Publishing Inc. This work is licensed under the Creative Commons Attribution International License (CC BY 4.0).

http://creativecommons.org/licenses/by/4.0/

\begin{abstract}
A path $\pi=\left[v_{1}, v_{2}, \cdots, v_{k}\right]$ in a graph $G=(V, E)$ is an uphill path if $\operatorname{deg}\left(v_{i}\right) \leq \operatorname{deg}\left(v_{i+1}\right)$ for every $1 \leq i \leq k$. A subset $S \subseteq V(G)$ is an uphill dominating set if every vertex $v_{i} \in V(G)$ lies on an uphill path originating from some vertex in $S$. The uphill domination number of $G$ is denoted by $\gamma_{u p}(G)$ and is the minimum cardinality of the uphill dominating set of $G$. In this paper, we introduce the uphill domination polynomial of a graph $G$. The uphill domination polynomial of a graph $G$ of $n$ vertices is the polynomial $U P(G, x)=\sum_{i=\gamma_{u p}(G)}^{n} u p(G, i) x^{i}$, where $u p(G, i)$ is the number of uphill dominating sets of size $i$ in $G$, and $\gamma_{u p}(G)$ is the uphill domination number of $G$, we compute the uphill domination polynomial and its roots for some families of standard graphs. Also, $U P(G, x)$ for some graph operations is obtained.
\end{abstract}

\section{Keywords}

Domination, Uphill Domination, Uphill Domination Polynomial

\section{Introduction}

In this paper, we are concerned with simple graphs which are finite, undirected with no loops nor multiple edges. Throughout this paper, we let $|V(G)|=n$ and $|E(G)|=m$. In a graph $G=(V, E)$, the degree of $v \in V(G)$ denoted by $\operatorname{deg}(v)$ is the number of edges that incident with $v$. A path in $G$ is an alternating sequence of distinct vertices. A path is an uphill path if for every $1 \leq i \leq k$ we have $\operatorname{deg}\left(v_{i}\right) \leq \operatorname{deg}\left(v_{i+1}\right) \quad[1]$.

The bistar graph $S_{k_{1}, k_{1}}$ with $n=2 k_{1}+2$ vertices is obtained by joining the non-pendant vertices of two copies of star graph $S_{k_{1}}$ by new edge. The corona of two graphs $G_{1}$ and $G_{2}$ with $n_{1}$ and $n_{2}$ vertices, respectively, denoted by 
$G=G_{1} \circ G_{2}$ is obtained by taking one copy of $G_{1}$ and $n_{1}$ copies of $G_{2}$ and joining the $i$ th vertex of $G_{1}$ with an edge to every vertex in the $i$ th copy of $G_{2}$. The corona $G \circ K_{1}$ (in particular) is the graph constructed by a copy of $G$, where for each vertex $v \in V(G)$ a new vertex $v^{\prime}$ and a pendant edge $v v^{\prime}$ are added. The tadpole graph $T_{s, k}$ is a graph consisting of a cycle graph $C_{s}$ on at least three vertices and a path graph $P_{k}$ on $k$ vertices connected with bridge. The wheel graph $W_{s}$ is a graph formed by connecting a single vertex to all vertices of a cycle graph $C_{s}$. The book graph is a Cartesian product $B_{m}=S_{m} \times P_{2}$, where $S_{m}$ is the star graph with $m+1$ vertices and $P_{2}$ is the path graph on two vertices. Also, the windmill graph $W d(s, k)$ is a graph constructed for $s \geq 2$ and $k \geq 2$ by joining $k$ copies of the complete graph $K_{s}$ at a shared universal vertex. The dutch windmill graph $D(s, k)$ is the graph obtained by taking $k$ copies of the cycle graph $C_{s}$ with a vertex in common. Also, the friendship $F_{k}$ is a graph that constructed by joining $k$ copies of the cycle graph $C_{3}$ and observes that $F_{k}$ is a special case of $D(s, k)$. Finlay, the firefly graph $F_{s, t, k}$ with $s, t, k \geq 0$ and $n=2 s+2 t+k+1$ vertices is defined by consisting of $s$ triangles, $t$ pendent paths of length 2 and $k$ pendent edges, sharing a common vertex. Any terminology not mentioed here we refer the reader to [2].

A set $S \subseteq V$ of vertices in a graph $G$ is called a dominating set if every vertex $v \in V$ is either $v \in S$ or $v$ is adjacent to an element of $S$, The uphill dominating set "UDS" is a set $S \subseteq V$ having the property that every vertex $v \in V$ lies on an uphill path originating from some vertex in $S$. The uphill domination number of a graph $G$ is denoted by $\gamma_{u p}(G)$ and is defined to be the minimum cardinality of the UDS of $G$. Moreover, it's customary to denote the UDS having the minimum cardinality by $\gamma_{u p}(G)$-set, for more details in domination see [3] and [4].

Representing a graph by using a polynomial is one of the algebraic representations of a graph to study some of algebraic properties and graph's structure. In general graph polynomials are a well-developed area which is very useful for analyzing properties of the graphs.

The domination polynomial [5] and the uphill domination of a graph [6], motivated us to introduce and study the uphill domination polynomial and the uphill domination roots of a graph.

\section{Uphill Domination Polynomial}

Definition 2.1. For any graph $G$ of $n$ vertices, the uphill domination polynomial of $G$ is defined by

$$
U P(G, x)=\sum_{i=\gamma_{u p}(G)}^{n} u p(G, i) x^{i},
$$

where $u p(G, i)$ is the number of uphill dominating sets of size $i$ in $G$. The set of roots of $\operatorname{UP}(G, x)$ is called uphill domination roots of graph $G$ and denoted by $Z_{\text {up }}(G)$.

Example 2.2. The uphill domination polynomial of House graph $H$ (as shown 
in Figure 1) with 6 vertices and $\gamma_{u p}(H)=2$ is given by $U P(H, x)=2 x^{2}+7 x^{3}+9 x^{4}+5 x^{5}+x^{6}$. Furthermore, $Z_{u p}(H)=\{0,-1,-2\}$.

The following theorem gives the sufficient condition for the uphill domination polynomial of $r$-regular graph.

Theorem 2.3. Let $G$ be connected graph with $n \geq 2$ vertices. Then, $U P(G, x)=(1+x)^{n}-1$ if and only if $G$ is $r$-regular graph.

Proof. Let $G$ be a connected graph of $n \geq 2$ vertices. Suppose that the uphill domination polynomial of $G$ is given by

$$
U P(G, x)=(1+x)^{n}-1=n x+\left(\begin{array}{l}
n \\
2
\end{array}\right) x^{2}+\cdots+x^{n} .
$$

Since the first coefficient of the polynomial is $n$, then it is easily verified that for every $v \in V(G)$, the singleton vertex set $\{v\}$ is an UDS in $G$. Assume that $G$ is not $r$-regular graph. Hence there exists a vertex $u \in V(G)$ such that $\operatorname{deg}(u)=s \neq r$. Now, we have two cases:

Case 1: If $s>r$, then the set $\{u\}$ is not UDS which contradict that every singleton vertex set is an UDS in $G$.

Case 2: If $s<r$, then for all $u \neq v$ with $\operatorname{deg}(v)=r$, we get the set $\{v\}$ is not UDS which is also contradict that every singleton vertex set is an UDS in $G$.

Thus, $G$ must be $r$-regular graph.

On the other hand, suppose that $G$ is $r$-regular graph with $n \geq 2$ vertices. We have $\gamma_{u p}(G)=1$, then there exist $n$ UDS of size one, while for $i=2$ there are $\left(\begin{array}{l}n \\ 2\end{array}\right)$ UDS and so on. Thus, we can write the uphill domination polynomial as

$$
U P(G, x)=n x+\left(\begin{array}{l}
n \\
2
\end{array}\right) x^{2}+\left(\begin{array}{l}
n \\
2
\end{array}\right) x^{3}+\cdots+\left(\begin{array}{l}
n \\
n
\end{array}\right) x^{n}=(1+x)^{n}-1 .
$$

Corollary 2.4. Let $G$ ba a graph with $s$ vertices. If $G$ is a cycle $C_{s}$ or complete graph $K_{s}$, then $U P(G, x)=(1+x)^{s}-1$.

Corollary 2.5. The uphill domination polynomial for the regular graph $G=C_{s} \times C_{k}$ with sk vertices is given by $\operatorname{UP}(G, x)=(1+x)^{s k}-1$.

Corollary 2.6. [6] Let $G$ be a graph with $m$ components. Then,

$$
\gamma_{u p}(G)=\sum_{j=1}^{m} \gamma_{u p}\left(G_{i}\right) \text {. }
$$

Proposition 2.7. If a graph $G$ with $n$ vertices consists of $m$ components $G_{1}, G_{2}, \cdots, G_{m}$, then

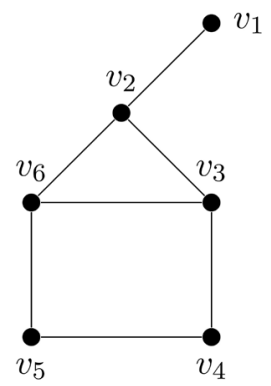

Figure 1. The House graph. 


$$
U P(G, x)=\prod_{i=1}^{m} U P\left(G_{i}, x\right) .
$$

Proof. By using mathematical induction we found that for $m=1$ the statement is true and the proof is trivial. Suppose that the statement is true when $m=k$ such that

$$
U P(G, x)=\prod_{i=1}^{k} U P\left(G_{i}, x\right) .
$$

Now, we prove that the statement is true when $m=k+1$. Let $G$ consists of $k+1$ components that mean $G=G_{1} \cup G_{2} \cup \cdots \cup G_{k+1}$. If the set $\left\{r_{1}, r_{2}, \cdots, r_{k+1}\right\}$ represent the uphill domination number for the components of $G$ respectively, such that $\gamma_{u p}\left(G_{i}\right)=r_{i} \quad \forall 1 \leq i \leq k+1$. Then, by Corollary (2.6) it easily to see that

$$
\gamma_{u p}(G)=\gamma_{u p}\left(\bigcup_{1 \leq i \leq k+1} G_{i}\right)=\sum_{1 \leq i \leq k+1} \gamma_{u p}\left(G_{i}\right)=r_{1}+\cdots+r_{k+1}=r .
$$

Thus, $u p(G, r)$ is exactly equal the number of way for choosing an UDS of size $r_{1}$ in $G_{1}$ and an UDS of size $r_{2}$ in $G_{2}$ and so on. Hence, $u p(G, r)$ is the coefficient of $x^{r}$ in $U P\left(G_{1}, x\right) U P\left(G_{2}, x\right) \cdots U P\left(G_{k+1}, x\right)$ and in $U P(G, x)$. In the same argument we can proof for all $u p(G, j)$, where $r \leq j \leq n$ that

$$
u p(G, j)=u p\left(G_{1}, j\right) \cdots u p\left(G_{k+1}, j\right)=\prod_{i=1}^{k+1} u p\left(G_{i}, j\right) \text {. }
$$

Thus, for $m=k+1$ the statement is true and the proof is done.

Theorem 2.8. For any path $P_{n}$ with $n \geq 3$ vertices, $U P(G, x)=x^{2}(1+x)^{n-2}$. Furthermore, $Z_{u p}\left(P_{n}\right)=\{0,-1\}$.

Proof. Let $G$ be a path graph $P_{n}$ with $n \geq 3$. We know that $\gamma_{u p}\left(P_{n}\right)=2$, then there is only one UDS of size two. For $i=3$ there are $n-2$ UDS of size three and so on. Thus, we get

$$
\begin{aligned}
U P(G, x) & =x^{2}+\left(\begin{array}{c}
n-2 \\
1
\end{array}\right) x^{3}+\left(\begin{array}{c}
n-2 \\
2
\end{array}\right) x^{4}+\cdots+\left(\begin{array}{c}
n-2 \\
n-2
\end{array}\right) x^{n} \\
& =x^{2}\left[\begin{array}{c}
\left.1+\sum_{i=1}^{n-2}\left(\begin{array}{c}
n-2 \\
i
\end{array}\right) x^{i}\right] \\
\end{array}=x^{2}\left[\sum_{i=0}^{n-2}\left(\begin{array}{c}
n-2 \\
i
\end{array}\right) x^{i}\right]\right. \\
& =x^{2}(1+x)^{n-2} .
\end{aligned}
$$

Theorem 2.9. For any graph $G$. $U P(G, x)=x^{n}$ if and only if $G \cong \bar{K}_{n}$.

Proof. Let $G$ be a graph with $U P(G, x)=x^{n}$. Since, $U P\left(\bar{K}_{1}, x\right)=x$, then we can write that

$$
\begin{aligned}
U P(G, x) & =x^{n} \\
& =\underbrace{x \cdot x \cdots x}_{n \text { times }} \\
& =\underbrace{U P\left(\bar{K}_{1}, x\right) \cdot U P\left(\bar{K}_{1}, x\right) \cdots U P\left(\bar{K}_{1}, x\right)}_{n \text { times }} \\
& =U P\left(\bar{K}_{n}, x\right) .
\end{aligned}
$$


Thus, $G \cong \bar{K}_{n}$. On the other hand if $G \cong \bar{K}_{n}$, then by Proposition (2.7) we get $U P(G, x)=x^{n}$.

Corollary 2.10. A graph $G$ has one uphill domination root if and only if $G \cong \bar{K}_{n}$.

Theorem 2.11. Let $G$ be a bistar graph $S_{k_{1}, k_{1}}$ with $n=2 k_{1}+2$ vertices. Then, $U P(G, x)=x^{2 k_{1}}(1+x)^{2}$. Furthermore, $Z_{u p}(G)=\{0,-1\}$.

Proof. Let $G$ be a bistar graph $S_{k_{1}, k_{1}}$ with $n=2 k_{1}+2$ vertices, we have $\gamma_{\text {up }}(G)=2 k_{1}$. Then, there is only one UDS of size $2 k_{1}$ and for $i=2 k_{1}+1$ there are two UDS. Finally, for $i=2 k_{1}+2=n$ there is only one UDS. Thus, the result will be as following

$$
\begin{aligned}
U P(G, x) & =x^{2 k_{1}}+2 x^{2 k_{1}+1}+x^{2 k_{1}+2} \\
& =x^{2 k_{1}}\left[1+2 x+x^{2}\right] \\
& =x^{2 k_{1}}(1+x)^{2} .
\end{aligned}
$$

Theorem 2.12. For any graph $G \cong K_{r, s}$ with $r<s$ and $r+s \geq 3$ vertices, $U P(G, x)=x^{s}(1+x)^{r}$. Furthermore, $Z_{u p}\left(K_{r, s}\right)=\{0,-1\}$.

Proof. Let $G$ is a complete bipartite graph $K_{r, s}$ with $r<s$, then we have $\gamma_{u p}\left(K_{r, s}\right)=s$. There is only one UDS of size $s$. Now, for $i=s+1$ there exist $r$ UDS. For $i=s+2$ there exist $\left(\begin{array}{l}r \\ 2\end{array}\right)$ UDS and so on. Thus, we get

$$
\begin{aligned}
U P(G, x) & =x^{s}+\left(\begin{array}{l}
r \\
1
\end{array}\right) x^{s+1}+\left(\begin{array}{l}
r \\
2
\end{array}\right) x^{s+2}+\cdots+\left(\begin{array}{l}
r \\
r
\end{array}\right) x^{s+r} \\
& =x^{s}+\sum_{i=1}^{r}\left(\begin{array}{l}
r \\
i
\end{array}\right) x^{s+i} \\
& =x^{s}\left[\sum_{i=0}^{r}\left(\begin{array}{l}
r \\
i
\end{array}\right) x^{i}\right] \\
& =x^{s}(1+x)^{r} .
\end{aligned}
$$

Corollary 2.13. For any graph $G \cong S_{r}$ with $r+1$ vertices, $U P(G, x)=x^{r}(1+x)$. Furthermore, $Z_{u p}(G)=\{0,-1\}$.

The generalization of Theorem 0.12 is the following result.

Theorem 2.14. For any graph $G \cong K_{r_{1}, \cdots, r_{k}}$ where $r_{1}<r_{2}<\cdots<r_{k}$ with $n=\sum_{i=1}^{k} r_{i}$ vertices, $U P(G, x)=x^{r_{k}}(1+x)^{n-r_{k}}$. Furthermore, $Z_{u p}\left(K_{r_{1}, \cdots, r_{k}}\right)=\{0,-1\}$.

Proof. Let $G$ be a complete $k$-partite graph $K_{r_{1}, \cdots, r_{k}}$ with $r_{1}<r_{2}<\cdots<r_{k}$, we have $\gamma_{u p}\left(K_{r_{1}, \cdots, r_{k}}\right)=r_{k}$. There is only one UDS of size $r_{k}$ for $i=r_{k}+1$ there are $n-r_{k}$ UDS of size $r_{k}+1$. Also, for $i=r_{k}+2$ there are $\left(\begin{array}{c}n-r_{k} \\ 2\end{array}\right)$ and so on. Thus, 


$$
\begin{aligned}
U P(G, x) & =x^{r_{k}}+\left(\begin{array}{c}
n-r_{k} \\
1
\end{array}\right) x^{r_{k}+1}+\left(\begin{array}{c}
n-r_{k} \\
2
\end{array}\right) x^{r_{k}+2}+\cdots+\left(\begin{array}{c}
n-r_{k} \\
n-r_{k}
\end{array}\right) x^{n} \\
& =x^{r_{k}}+\sum_{i=1}^{n-r_{k}}\left(\begin{array}{c}
n-r_{k} \\
i
\end{array}\right) x^{r_{k}+i} \\
& =x^{r_{k}}\left[\sum_{i=0}^{n-r_{k}}\left(\begin{array}{c}
n-r_{k} \\
i
\end{array}\right) x^{i}\right] \\
& =x^{r_{k}}(1+x)^{n-r_{k}} .
\end{aligned}
$$

Proposition 2.15. For any graph $G \cong K_{r_{1}, r_{2}, \cdots, r_{k}}$ with $n=\sum_{i=1}^{k} r_{i}$ vertices we have the following:

1) If $r_{1} \leq r_{2} \leq \cdots \leq r_{k-1}<r_{k}$, such that at least two partite sets of the same size, then $U P(G, x)=x^{r_{k}}(1+x)^{n-r_{k}}$.

2) If $r_{1}=r_{2}=\cdots=r_{k}$, then the graph is regular and $U P(G, x)=(1+x)^{n}-1$.

Theorem 2.16. For any graph $G \cong K_{r_{1}, r_{2}, \cdots, r_{k}}$ with $n=\sum_{i=1}^{k} r_{i}$ vertices, where $r_{1} \leq r_{2} \leq \cdots<r_{k-1}=r_{k}$. Then,

$$
U P(G, x)=\sum_{h=1}^{n}\left[\sum_{\substack{r_{1} \geq 1 \\
r_{1}+r_{2}=h}}\left(\begin{array}{c}
2 r_{k} \\
r_{1}
\end{array}\right)\left(\begin{array}{c}
n-2 r_{k} \\
r_{2}
\end{array}\right)\right] x^{h} .
$$

Proof. Let $G$ be a complete $k$-partite graph $K_{r_{1}, \cdots, r_{k}}$ with $r_{1} \leq r_{2} \leq \cdots<r_{k-1}=r_{k}$, then we have $\gamma_{u p}\left(K_{r_{1}, \cdots, r_{k}}\right)=1$. Let divide the vertices of a graph into two sets $R_{1}$ and $R_{2}$ where $R_{1}$ contains the vertices of $r_{k}$ and $r_{k-1}$ which means $R_{1}$ is of cardinality $2 r_{k}$ while $R_{2}=V(G) \backslash R_{1}$ this implies that $R_{2}$ is of cardinality $n-2 r_{k}$. Thus, we get

$$
u p(G, 1)=\left(\begin{array}{c}
2 r_{k} \\
1
\end{array}\right)\left(\begin{array}{c}
n-2 r_{k} \\
0
\end{array}\right)=2 r_{k} .
$$

We have for up $(G, 2)$,

$$
u p(G, 2)=\left(\begin{array}{c}
2 r_{k} \\
2
\end{array}\right)\left(\begin{array}{c}
n-2 r_{k} \\
0
\end{array}\right)+\left(\begin{array}{c}
2 r_{k} \\
1
\end{array}\right)\left(\begin{array}{c}
n-2 r_{k} \\
1
\end{array}\right) \text {. }
$$

Also, for $u p(G, 3)$ we get

$$
u p(G, 3)=\left(\begin{array}{c}
2 r_{k} \\
3
\end{array}\right)\left(\begin{array}{c}
n-2 r_{k} \\
0
\end{array}\right)+\left(\begin{array}{c}
2 r_{k} \\
2
\end{array}\right)\left(\begin{array}{c}
n-2 r_{k} \\
1
\end{array}\right)+\left(\begin{array}{c}
2 r_{k} \\
1
\end{array}\right)\left(\begin{array}{c}
n-2 r_{k} \\
2
\end{array}\right)
$$

And so on we get for all $u p(G, h)$, where $1 \leq h \leq n$

$$
u p(G, h)=\sum_{\substack{r_{1} \geq 1 \\
r_{1}+r_{2}=h}}\left(\begin{array}{c}
2 r_{k} \\
r_{1}
\end{array}\right)\left(\begin{array}{c}
n-2 r_{k} \\
r_{2}
\end{array}\right) \text {. }
$$

Thus, the proof is done.

Theorem 2.17. For any graph $G \cong W_{s}$ with $s+1$ vertices and $s>3$, then $U P(G, x)=(1+x)\left[(1+x)^{s}-1\right]$.

Proof. Let $G$ be a wheel graph $W_{s}(s>3)$, then we have $\gamma_{u p}\left(W_{s}\right)=1$. There 
are $s$ UDS of size one. For $i=2$ there are $\left(\begin{array}{c}s+1 \\ 2\end{array}\right)$ UDS of size two and so on. Thus,

$$
\begin{aligned}
U P(G, x) & =s x+\left(\begin{array}{c}
s+1 \\
2
\end{array}\right) x^{2}+\left(\begin{array}{c}
s+1 \\
3
\end{array}\right) x^{3}+\cdots+\left(\begin{array}{c}
s+1 \\
s+1
\end{array}\right) x^{s+1} \\
& =\left[\sum_{i=0}^{s+1}\left(\begin{array}{c}
s+1 \\
i
\end{array}\right) x^{i}\right]-(x+1) \\
& =(x+1)^{s+1}-(x+1) \\
& =(x+1)\left[(x+1)^{s}-1\right] .
\end{aligned}
$$

Corollary 2.18. For any wheel graph $W_{s}$ and $s>3$ we have

$$
Z_{u p}\left(W_{s}\right)= \begin{cases}\{0,-1,-2\}, & \text { if } s \text { is even. } \\ \{0,-1\}, & \text { if } s \text { is odd. }\end{cases}
$$

\section{Uphill Domination Polynomials of Graphs under Some Binary Operations}

Theorem 3.1. Let $G \cong P_{r} \times P_{s}$ be a grid graph with $r s$ vertices and $r, s \geq 4$. Then, $U P(G, x)=x^{4}(1+x)^{r s-4}$.

Proof. Let $G$ be a grid graph with $r s$ vertices and $r, s \geq 4$, then we have $\gamma_{u p}(G)=4$. Note that, there is only one UDS of size four. For $i=5$, there are $r s-4$ UDS of size five and so on. Thus, we get

$$
\begin{aligned}
U P(G, x) & =x^{4}+\left(\begin{array}{c}
r s-4 \\
1
\end{array}\right) x^{5}+\cdots+\left(\begin{array}{c}
r s-4 \\
r s-4
\end{array}\right) x^{r s} \\
& =x^{4}\left[\sum_{i=0}^{r s-4}\left(\begin{array}{c}
r s-4 \\
i
\end{array}\right) x^{i}\right] \\
& =x^{4}(1+x)^{r s-4} .
\end{aligned}
$$

Theorem 3.2. Let $G \cong C_{r} \circ \bar{K}_{s}$ be a corona graph with $r s+r$ vertices. Then, $U P(G, x)=x^{r s}(1+x)^{r}$.

Proof. Let $G \cong C_{r} \circ \bar{K}_{s}$ with $r s+r$ vertices, we have $\gamma_{u p}\left(C_{r} \circ \bar{K}_{s}\right)=r s$. For $r s$ vertices, there is only one UDS of size $r s$. For $r s+1$ vertices, there are $r$ UDS and so on. Thus, we get

$$
\begin{aligned}
U P(G, x) & =x^{r s}+\left(\begin{array}{l}
r \\
1
\end{array}\right) x^{r s+1}+\cdots+\left(\begin{array}{l}
r \\
r
\end{array}\right) x^{r s+r} \\
& =\sum_{i=0}^{r}\left(\begin{array}{l}
r \\
i
\end{array}\right) x^{r s+i} \\
& =x^{r s}\left[\sum_{i=0}^{r}\left(\begin{array}{l}
r \\
i
\end{array}\right) x^{i}\right] \\
& =x^{r s}(1+x)^{r} .
\end{aligned}
$$

Corollary 3.3. Let $G \cong C_{r} \circ K_{1}$ be a corona graph with $2 r$ vertices. Then, $U P(G, x)=x^{r}(1+x)^{r}$. 
Theorem 3.2 can generalize in the following result.

Theorem 3.4. For any nontrivial connected graph $H$ with $r$ vertices, if $G \cong H \circ \bar{K}_{s}$, then, $U P(G, x)=x^{r s}(1+x)^{r}$.

Proof. The proof similarly to the proof of Theorem 3.2.

Theorem 3.5. Let $G$ be a book graph $B_{m}=P_{2} \times S_{m}$ with $2 m+2$ vertices. Then,

$$
\begin{aligned}
U P(G, x)= & 2^{m} x^{m}+\left[m\left(2^{m-1}\right)+2^{m+1}\right] x^{m+1} \\
& +\sum_{i=2}^{2 m-1}\left[\left(\begin{array}{c}
m \\
i
\end{array}\right) 2^{m-i}+\left(\begin{array}{c}
m \\
i-1
\end{array}\right) 2^{m-i+2}+\left(\begin{array}{c}
m \\
i-2
\end{array}\right) 2^{m-i+2}\right] x^{m+i} \\
& +\left[1+m 2^{2}+\left(\begin{array}{c}
m \\
2
\end{array}\right) 2^{2}\right] x^{2 m}+(2 m+2) x^{2 m+1}+x^{2 m+2} .
\end{aligned}
$$

Proof. Suppose we have the book graph $B_{m}=P_{2} \times S_{m}$ with $2 m+2$ vertices, then we have $\gamma_{u p}\left(P_{2} \times S_{m}\right)=m$. Let divide the vertices of $B_{m}$ into $m+1$ sets "as shown in Figure 2" let the set $R_{i}=\left\{u_{i}, v_{i}\right\}$ i.e., $1 \leq i \leq m$ while $R_{m+1}=\{u, v\}$. Since $\gamma_{u p}\left(P_{2} \times S_{m}\right)=m$, then for $u p(G, m)$ we have to take one vertex from each $R_{i}(i \neq m+1)$ so, there exist $2^{m}$ UDS of size $m$. For up $(G, m+1)$ we have,

$$
\begin{aligned}
u p(G, m+1) & =\underbrace{\left(\begin{array}{l}
2 \\
1
\end{array}\right) \ldots\left(\begin{array}{l}
2 \\
1
\end{array}\right)}_{(m+1) \text { times }}+\sum_{\sum_{r_{1}} \cdots, r_{m} \geq 1}\left(\begin{array}{l}
2 \\
r_{1}
\end{array}\right) \ldots\left(\begin{array}{l}
2 \\
r_{m}
\end{array}\right)\left(\begin{array}{l}
2 \\
0
\end{array}\right) \\
& =2^{m+1}+m\left(2^{m-1}\right) .
\end{aligned}
$$

Also, for $u p(G, m+2)$ we get

$$
\begin{aligned}
u p(G, m+2)= & \sum_{\substack{\sum_{r_{i}}=m+2 \\
r_{1} \cdots, r_{m} \geq 1}}\left(\begin{array}{l}
2 \\
r_{1}
\end{array}\right) \ldots\left(\begin{array}{c}
2 \\
r_{m}
\end{array}\right)\left(\begin{array}{l}
2 \\
0
\end{array}\right)+\sum_{\substack{\sum_{r_{i}}=m+1 \\
r_{1} \cdots, r_{m} \geq 1}}\left(\begin{array}{l}
2 \\
r_{1}
\end{array}\right) \ldots\left(\begin{array}{c}
2 \\
r_{m}
\end{array}\right)\left(\begin{array}{l}
2 \\
1
\end{array}\right) \\
& +\sum_{\substack{\sum_{1} r_{i}=m \\
r_{1} \cdots, r_{m} \geq 1}}\left(\begin{array}{c}
2 \\
r_{1}
\end{array}\right) \ldots\left(\begin{array}{c}
2 \\
r_{m}
\end{array}\right)\left(\begin{array}{l}
2 \\
2
\end{array}\right) \\
= & \left(\begin{array}{c}
m \\
2
\end{array}\right) 2^{m-2}+\left(\begin{array}{c}
m \\
1
\end{array}\right) 2^{m}+\left(\begin{array}{c}
m \\
0
\end{array}\right) 2^{m} \\
= & \left(\begin{array}{c}
m \\
2
\end{array}\right) 2^{m-2}+m 2^{m}+2^{m} .
\end{aligned}
$$

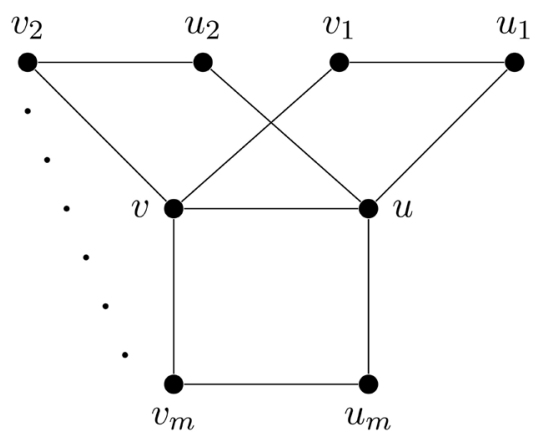

Figure 2. A Book Graph $B_{m}$. 
Therefore, for $u p(G, m+3)$ we have

$$
\begin{aligned}
& u p(G, m+3)=\sum_{\substack{\sum_{r_{1}=m+3}=r_{m} \geq 1 \\
r_{1}}}\left(\begin{array}{l}
2 \\
r_{1}
\end{array}\right) \ldots\left(\begin{array}{c}
2 \\
r_{m}
\end{array}\right)\left(\begin{array}{l}
2 \\
0
\end{array}\right)+\sum_{\substack{\sum_{r_{1}}=m+2 \\
r_{1} \cdots, r_{m} \geq 1}}\left(\begin{array}{l}
2 \\
r_{1}
\end{array}\right) \ldots\left(\begin{array}{c}
2 \\
r_{m}
\end{array}\right)\left(\begin{array}{l}
2 \\
1
\end{array}\right) \\
& +\sum_{\substack{\sum_{1}=m+1 \\
r_{1}=r_{m} \geq 1}}\left(\begin{array}{l}
2 \\
r_{1}
\end{array}\right) \cdots\left(\begin{array}{c}
2 \\
r_{m}
\end{array}\right)\left(\begin{array}{l}
2 \\
2
\end{array}\right) \\
& =\left(\begin{array}{c}
m \\
3
\end{array}\right) 2^{m-3}+\left(\begin{array}{c}
m \\
2
\end{array}\right) 2^{m}+\left(\begin{array}{c}
m \\
1
\end{array}\right) 2^{m} \\
& =\left(\begin{array}{c}
m \\
3
\end{array}\right) 2^{m-3}+\left(\begin{array}{c}
m \\
2
\end{array}\right) 2^{m}+m 2^{m} \text {. }
\end{aligned}
$$

And so on, we use the same argument until up $(G, 2 m-1)$. After that, for $u p(G, 2 m)$ we have

$$
\begin{aligned}
u p(G, 2 m)= & \left(\begin{array}{l}
2 \\
2
\end{array}\right) \ldots\left(\begin{array}{l}
2 \\
2
\end{array}\right)\left(\begin{array}{l}
2 \\
0
\end{array}\right)+\sum_{\substack{\sum_{r_{i}}=2 m-1 \\
r_{1} \ldots, r_{m} \geq 1}}\left(\begin{array}{l}
2 \\
r_{1}
\end{array}\right) \ldots\left(\begin{array}{c}
2 \\
r_{m}
\end{array}\right)\left(\begin{array}{l}
2 \\
1
\end{array}\right) \\
& +\sum_{\substack{\sum_{r_{i}}=2 m-2 \\
r_{1} \cdots, r_{m} \geq 1}}\left(\begin{array}{l}
2 \\
r_{1}
\end{array}\right) \ldots\left(\begin{array}{c}
2 \\
r_{m}
\end{array}\right)\left(\begin{array}{l}
2 \\
2
\end{array}\right) \\
= & 1+m 2^{2}+\left(\begin{array}{c}
m \\
2
\end{array}\right) 2^{2} .
\end{aligned}
$$

Finally,

$$
u p(G, 2 m+1)=\left(\begin{array}{c}
2 m+2 \\
2 m+1
\end{array}\right)=2 m+2 \quad \& \quad u p(G, 2 m+2)=1
$$

Thus, the proof is completed.

Theorem 3.6. Let $G$ be a graph. If $G \cong P_{k} \times C_{s}$ with sk vertices, then

$$
U P(G, x)=\sum_{t=2}^{s k}\left[\sum_{\substack{r_{1}, r_{2} \geq 1 \\
r_{1}+r_{2}+r_{3}=t}}\left(\begin{array}{l}
s \\
r_{1}
\end{array}\right)\left(\begin{array}{c}
s \\
r_{2}
\end{array}\right)\left(\begin{array}{c}
s k-2 s \\
r_{3}
\end{array}\right)\right] x^{t} .
$$

Proof. Let $G \cong P_{k} \times C_{s}$ with sk vertices, then we have $\gamma_{u p}\left(P_{k} \times C_{s}\right)=2$. We first divide the vertices of $G$ into three sets called them $R_{1}, R_{2}$ and $R_{3}$, where $R_{1}$ (resp. $R_{2}$ ) is contains the vertices of the outer cycle (resp. inner cycle) which every vertex is of degree three. The third set $R_{3}$ contains the vertices of the middle cycles, where every vertex is of degree four. Note that, any UDS should contain at least one vertex form $R_{1}$ and one vertex from $R_{2}$. Thus, for $\operatorname{up}(G, 2)$

$$
u p(G, 2)=\left(\begin{array}{l}
s \\
1
\end{array}\right)\left(\begin{array}{l}
s \\
1
\end{array}\right)\left(\begin{array}{c}
s k-2 s \\
0
\end{array}\right)=s^{2}
$$

For $\operatorname{up}(G, 3)$ we have

$$
u p(G, 3)=\sum_{\substack{r_{1}, r_{2} \geq 1 \\
r_{1}+r_{2}+r_{3}=3}}\left(\begin{array}{c}
s \\
r_{1}
\end{array}\right)\left(\begin{array}{c}
s \\
r_{2}
\end{array}\right)\left(\begin{array}{c}
s k-2 s \\
r_{3}
\end{array}\right) .
$$

And so on, we use the same argument for all $u p(G, t)$ i.e., $3 \leq t \leq s k$ and 
the proof is done.

Theorem 3.7. Let $G$ ba a tadpole graph $T_{s, k}$ with $s+k$ vertices. Then,

$$
U P(G, x)=(s-1) x^{2}+\sum_{t=3}^{s+k}\left[\sum_{\substack{r_{1}+r_{2}=t-1 \\
r_{2} \geq 1}}\left(\begin{array}{c}
k \\
r_{1}
\end{array}\right)\left(\begin{array}{c}
s-1 \\
r_{2}
\end{array}\right)\right] x^{t} .
$$

Proof. Let $G$ be a tadpole graph $T_{s, k}$ with $s+k$ vertices, we have $\gamma_{u p}\left(T_{s, k}\right)=2$. We first divide the vertices of $T_{s, k}$ into three sets called them $R_{1}, R_{2}$ and $R_{3}$ such that $R_{1}$ is a singleton set that contains the pendant vertex, $R_{2}$ has $k$ vertices each of them is of degree two except one vertex is of degree three while the last set $R_{3}$ has $s-1$ vertices each of them of degree two which are the vertices that lies in a cycle part of a graph. Notice that, any UDS of $T_{s, k}$ should contains the pendant vertex and at least one vertex from $R_{3}$. Now, for up $(G, 2)$ we have to take the pendant vertex with one vertex from $R_{3}$, so there exist $s-1$ UDS of size two. For $u p(G, 3)$ we get

$$
u p(G, 3)=\sum_{\substack{r_{3} \geq 1 \\
r_{2}+r_{3}=2}}\left(\begin{array}{c}
k \\
r_{2}
\end{array}\right)\left(\begin{array}{c}
s-1 \\
r_{3}
\end{array}\right) \text {. }
$$

And so on, we use the same argument for all up(G,t) i.e., $3 \leq t \leq s+k$ and the proof is completed.

Theorem 3.8. Let $G$ be a windmill graph $W d(s, k)$ with $k(s-1)+1$ vertices. Then,

$$
U P(G, x)=(s-1)^{k} x^{k}+\sum_{t=k+1}^{k(s-1)+1}\left[\sum_{\substack{r_{1}, \cdots, r_{k} \geq 1 \\
r_{1}+\cdots+r_{k+1}=t}}\left(\begin{array}{c}
s-1 \\
r_{1}
\end{array}\right) \ldots\left(\begin{array}{c}
s-1 \\
r_{k}
\end{array}\right)\left(\begin{array}{c}
1 \\
r_{k+1}
\end{array}\right)\right] x^{t} .
$$

Proof. Let $G$ be a windmill graph with center vertex $w$, we have $\gamma_{u p}(G)=k$. Any minimum uphill domination set must contains one vertex from each copy of $K_{s}$ without the center vertex $w$, that means, we have $(s-1)^{k}$ uphill dominating set of size $k$. Suppose $R_{i}$ be the set of vertices of the i-th copy of $K_{S}$ without the center vertex $w$ and $R_{w}$ be the singleton, with the center vertex $w$. To get the number of uphill dominating sets of size $t=k+j$, where $j=1,2, \cdots,(k(s-2)+1)$, we need to select $r_{i}$ vertices from each $R_{i}$, and $r_{k+1}$ from $R_{w}$ where $i=1,2, \cdots, k, \quad \sum_{i=1}^{k+1} r_{i}=t$ and $r_{i} \geq 1$ for all $i=1,2, \cdots, k$. Hence,

$$
u p(G, t)=\sum_{\substack{r_{1}, \cdots, r_{k} \geq 1 \\
r_{1}+\cdots+r_{k+1}=t}}\left[\left(\begin{array}{c}
s-1 \\
r_{1}
\end{array}\right) \ldots\left(\begin{array}{c}
s-1 \\
r_{k}
\end{array}\right)\left(\begin{array}{c}
1 \\
r_{k+1}
\end{array}\right)\right]
$$

Thus,

$$
U P(G, x)=(s-1)^{k} x^{k}+\sum_{t=k+1}^{k(s-1)+1}\left[\sum_{\substack{r_{1}, \cdots, r_{k} \geq 1 \\
r_{1}+\cdots, r_{k+1}=t}}\left(\begin{array}{c}
s-1 \\
r_{1}
\end{array}\right) \ldots\left(\begin{array}{c}
s-1 \\
r_{k}
\end{array}\right)\left(\begin{array}{c}
1 \\
r_{k+1}
\end{array}\right)\right] x^{t} .
$$

Proposition 3.9. Let $G$ be a dutch windmill graph $D(s, k)$ with $s>3$ and $k(s-1)+1$ vertices. Then, 


$$
U P(D(s, k), x)=U P(W d(s, k), x) .
$$

Theorem 3.10. Let $G$ be a firefly graph $F_{s, t, k}$ with $s, t, k \geq 0$, $n=2 s+2 t+k+1$ vertices and $\gamma_{u p}(G)=s+t+k=b$. Then,

$$
\begin{aligned}
U p(G, x)= & 2^{s} x^{b}+\left[2^{s}(t+1)+2^{s-1}(s)\right] x^{b+1} \\
& +\sum_{h=b+2}^{n}\left[\sum_{\substack{r_{1}, \cdots, r_{2} \geq 1 \\
r_{1}+\cdots+r_{s+1}=h-(t+k)}}\left(\begin{array}{c}
s \\
r_{1}
\end{array}\right)\left(\begin{array}{c}
s \\
r_{2}
\end{array}\right) \ldots\left(\begin{array}{c}
s \\
r_{s}
\end{array}\right)\left(\begin{array}{c}
t+1 \\
r_{s+1}
\end{array}\right)\right] x^{h} .
\end{aligned}
$$

Proof. Let $G$ be a firefly graph $F_{s, t, k}$ with $n$ vertices and $\gamma_{u p}(G)=s+t+k=b$. First, let us divide the vertices of $G$ into $s+2$ sets and let $u$ be the shared vertex in $G$. Suppose that $R_{1} \subset V(G)$ contains the vertices of the first triangle without $u$, this implies $R_{1}$ has two vertices each of them are of degree two, also we mean by $R_{2} \subset V(G)$ the set that contains the vertices of the second triangle without $u$ and so on for all $R_{i}$, where $1 \leq i \leq s$. Now, the subset $R_{s+1} \subset V(G)$ contains $u$ in addition the $t$ vertices of the pendant paths that adjacent to $u$ which means $R_{s+1}$ is of cardinality $t+1$. Finally, $R_{s+2} \subset V(G)$ contains all the leaves vertices of $G$ which be exactly of cardinality $t+k$. Notice that, any UDS of $G$ should contain all the vertices of $R_{s+2}$ with at least one vertex from each $R_{i}$. Thus, for $u p(G, b)$ we have

$$
\begin{aligned}
u p(G, b) & =\sum_{\sum_{i=1}^{s+2} r_{i}=b}\left[\left(\begin{array}{l}
2 \\
r_{1}
\end{array}\right) \cdots\left(\begin{array}{c}
2 \\
r_{s}
\end{array}\right)\left(\begin{array}{c}
t+1 \\
r_{s+1}
\end{array}\right)\left(\begin{array}{c}
t+k \\
r_{s+2}
\end{array}\right)\right] \\
& =\left[\left(\begin{array}{l}
2 \\
1
\end{array}\right) \cdots\left(\begin{array}{l}
2 \\
1
\end{array}\right)\left(\begin{array}{c}
t+1 \\
0
\end{array}\right)\left(\begin{array}{c}
t+k \\
t+k
\end{array}\right)\right] \\
& =\underbrace{2 \times 2 \times \cdots \times 2}_{s \text { times }}=2^{s} .
\end{aligned}
$$

For $u p(G, b+1)$ we get

$$
\begin{aligned}
u p(G, b+1) & =\sum_{\sum_{i=1}^{s+2} r_{i}=b+1}\left[\left(\begin{array}{l}
2 \\
r_{1}
\end{array}\right) \ldots\left(\begin{array}{l}
2 \\
r_{s}
\end{array}\right)\left(\begin{array}{c}
t+1 \\
r_{s+1}
\end{array}\right)\left(\begin{array}{c}
t+k \\
t+k
\end{array}\right)\right] \\
& =\sum_{\sum_{i=1}^{s+1} r_{i}=(b+1)-(t+k)}\left[\left(\begin{array}{l}
2 \\
r_{1}
\end{array}\right) \ldots\left(\begin{array}{c}
2 \\
r_{s}
\end{array}\right)\left(\begin{array}{c}
t+1 \\
0
\end{array}\right)\right]+\underbrace{\left(\begin{array}{l}
2 \\
1
\end{array}\right) \ldots\left(\begin{array}{c}
2 \\
1
\end{array}\right)}_{s \text { times }}\left(\begin{array}{c}
t+1 \\
1
\end{array}\right) \\
& =2^{s-1}(s)+2^{s}(t+1) .
\end{aligned}
$$

And for $u p(G, b+2)$ we have

$$
\begin{aligned}
u p(G, b+2) & =\sum_{\sum_{i=1}^{s+2} r_{i}=b+2}\left[\left(\begin{array}{l}
2 \\
r_{1}
\end{array}\right) \ldots\left(\begin{array}{l}
2 \\
r_{s}
\end{array}\right)\left(\begin{array}{c}
t+1 \\
r_{s+1}
\end{array}\right)\left(\begin{array}{c}
t+k \\
t+k
\end{array}\right)\right] \\
& =\sum_{\sum_{i=1}^{s+1} r_{i}=(b+2)-(t+k)}\left[\left(\begin{array}{l}
2 \\
r_{1}
\end{array}\right) \ldots\left(\begin{array}{c}
2 \\
r_{s}
\end{array}\right)\left(\begin{array}{c}
t+1 \\
r_{s+1}
\end{array}\right)\right] .
\end{aligned}
$$

In the same argument we can find all $u p(G, h)$, where $b+2 \leq h \leq n$ and the proof is completed.

Corollary 3.11. Let $G$ be a friendship graph $F_{k}$ with $2 k+1$ vertices. Then, 


$$
U P(G, x)=2^{k} x^{k}+\left[2^{k}+k 2^{k-1}\right] x^{k+1}+\sum_{t=k+2}^{2 k+1}\left[\sum_{\substack{r_{1}, \cdots, r_{k} \geq 1 \\
r_{1}+\cdots+r_{k+1}=t}}\left(\begin{array}{c}
2 \\
r_{1}
\end{array}\right) \ldots\left(\begin{array}{c}
2 \\
r_{k}
\end{array}\right)\left(\begin{array}{c}
1 \\
r_{k+1}
\end{array}\right)\right] x^{t}
$$

\section{Open Problems}

Finally, for feature work we state the following definition.

Definition 4.1. Two graphs $G$ and $H$ are said to be uphill-equivalent if $U P(G, x)=U P(H, x)$. The uphill-equivalence classes of $G$ noted by $[G]_{u p}=\{H: H$ is uphill-equivalent to $G\}$.

\section{Example 4.2.}

1) $\left[K_{n}\right]_{u p}=\{H: H$ is regular graph of $n$ vertices $\}$.

2) The windmill graph $W d(s, k)$ and Dutch windmill graph $D(s, k)$ are uphill-equivalent.

We state the following open problems for feature work:

1) which graphs have two distinct uphill domination roots?

2) which families of graphs have only real uphill domination roots?

3) which graphs satisfy $[G]_{u p}=\{G\}$ ?

4) determine the uphill-equivalence classes for some new families of graphs.

\section{Conflicts of Interest}

The authors declare no conflicts of interest regarding the publication of this paper.

\section{References}

[1] Deering, J. (2013) Uphill \& Downhill Domination in Graphs and Related Graph Parameters. Thesis, East Tennessee State University, Johnson.

[2] Balakrishnan, R. and Ranganathan, K. (2012) A Textbook of Graph Theory. Springer Science \& Business Media, New York. https://doi.org/10.1007/978-1-4614-4529-6

[3] Haynes, T.W., Hedetniemi, S.T. and Slater, P.J. (1998) Fundamentals of Domination in Graphs. Marcel Dekker, Inc., New York.

[4] Hedetniemi, S.T., Haynes, T.W., Jamieson, J.D. and Jamieson, W.B. (2014) Downhill Domination in Graphs. Discussiones Mathematicae, Graph Theory, 34, 603-612. https://doi.org/10.7151/dmgt.1760

[5] Alikhani, S. and Peng, Y.H. (2009) Introduction to Domination Polynomial of a Graph. Ars Combinatoria, 114. arXiv:0905.2251

[6] Alsalomy, T., Saleh, A., Muthana, N. and Al shammakh, W. On the Uphill Domination Number of Graphs. (Submitted) 